\title{
Phenolic compounds in minimally processed potato: Effect of cultivar, tuber storage time and thermal treatment
}

\section{Abstract}

Potato (Solanum tuberosum spp.) is worldwide consumed mostly as table potato, but due to lifestyle changes, production of minimally processed potato (MPP) became an important sector of the food industry. From the health point of view, phenolic compounds as potatoes constituents are desirable, but also responsible for undesirable browning of peeled and sliced potato. The most abundant are phenolic acids such as chlorogenic acid, which is a substrate for enzymatic oxidation in the presence of phenolase and is responsible for browning processes. Its concentration in tubers depends on cultivar type as well as storage, and thereby can influence on shelf life of MPP. The purpose of the present study was to evaluate the effect of two potato cultivars of different tuber age on the phenolic content of MPP during 8 days of storage followed by thermal treatment. Cultivars Birgit and Lady Claire were selected and stored for 1 and 4 months in warehouse $\left(8{ }^{\circ} \mathrm{C} / \mathrm{RH} 95 \%\right)$. Potatoes were washed, peeled, sliced and treated with $2 \%$ sodium ascorbate solution for 3 minutes. After draining, MPP samples were vacuum packed in PE/PA bags and stored for 1,5 and 8 days at $10{ }^{\circ} \mathrm{C}$. After storage, samples of raw, boiled and fried MPP were freeze dried and phenolic content was determined by UPLC MS/MS. In all potato samples chlorogenic acid, catechin and epicatechin were determined, where chlorogenic acid predominated (almost $97 \%$ ).

Generally, lower phenolic content was determined in cv. Birgit samples. Comparing cultivars upon tubers age, total phenolic content (as sum of all determined polyphenols) was lower after 4 months storage and ranged from $3.33 \mathrm{mg} / 100 \mathrm{~g}$ freeze dried sample (raw $\mathrm{cv}$. Birgit/ $\mathrm{s}^{\text {st }}$ day) to $7.27 \mathrm{mg} / 100 \mathrm{~g}$ (raw cv. Lady Claire $/ 8^{\text {th }}$ day). MPP's phenolic content in raw potatoes increased over the storage time, except in cv. Lady Claire potatoes stored for 1 month. Influence of thermal treatments on the MPP phenolic content showed decrease in boiled and fried MPP prepared from 1 month stored potato, while in MPP samples prepared from 4 months stored potato did not follow a clear trend. Phenolic content was lower in boiled potatoes compared to fried potatoes, regardless cultivar type, probably because phenolics are lost by cooking in water. The current study demonstrates that cv. Birgit is more suitable in minimal processing than cv. Lady Claire according to lower phenolic content.

\section{Conflict of Interest}

There is no conflict of interest. 